\section{B A Institute of \\ YK Business Administration \\ 六下 \\ Karachi \\ Leadership and Ideas for Tomorrow}

Business Review

Volume 14 Issue 1 January-June 2019

$1-1-2019$

\title{
An evaluation of the forecast performance of DSGE and VAR Models: The case of a developing country
}

Shahzad Ahmad

State Bank of Pakistan

Adnan Haider

Institute of Business Administration, Karachi, Pakistan

Follow this and additional works at: https://ir.iba.edu.pk/businessreview

Part of the Finance Commons, Management Sciences and Quantitative Methods Commons, and the Marketing Commons

\section{(c) (1)}

This work is licensed under a Creative Commons Attribution 4.0 International License.

\section{Recommended Citation}

Ahmad, S., \& Haider, A. (2019). An evaluation of the forecast performance of DSGE and VAR Models: The case of a developing country. Business Review, 14(1), 28-52. Retrieved from https://doi.org/10.54784/ 1990-6587.1023

This article is brought to you by iRepository for open access under the Creative Commons Attribution 4.0 License and is available at https://ir.iba.edu.pk/businessreview/vol14/iss1/3. For more information, please contact irepository@iba.edu.pk. 


\title{
An evaluation of the forecast performance of DSGE and VAR Models: The case of a developing country
}

\author{
Shahzad Ahmad • Adnan Haider
}

\begin{abstract}
This paper estimates a DSGE model and three versions of VAR models (VARX, BVARX and BVAR) to analyze forecasting performance of these models in context of Pakistan. VAR models and a medium-scale DSGE model are estimated using quarterly data (1980Q4-2017Q2). Expanding window recursive out-of-sample forecasts for GDP growth, call money rate, CPI inflation and percent change in exchange rate are generated and compared over the period 2009Q1-2017Q2. Forecasting performance is analyzed by the comparison of bias and root mean squared errors (RMSE). Analysis of forecasting performance over 1-8 quarters forecast horizon reveals that BVAR model provides relatively better forecast in case of GDP growth, interest rate and inflation while BVARX provides more accurate forecast in case of exchange rate. In case of GDP growth, inflation and exchange rate, forecasting performance of DSGE model considerably improves as forecasting horizon expands. For longer forecast horizons, divergence between DSGE and Bayesian VAR forecasts tends to disappear. This implies that DSGE model is more relevant for medium term forecasting rather than short term forecasting. Structural interpretation of DSGE forecast errors reveals that there has been unutilized growth potential in economic activity. This slack in economic activity might be attributable to unnecessarily high interest rate and overvalued exchange rate.
\end{abstract}

Keywords Forecasting · DSGE models · VAR models · Pakistan.

Shahzad Ahmad

State Bank of Pakistan and Institute of Business Administration, Karachi-Pakistan

E-mail: shahzad.ahmad@sbp.org.pk

Adnan Haider

Institute of Business Administration (IBA), Karachi-Pakistan 


\section{Introduction}

For successful conduct of forward looking monetary policy, two elements are of critical importance: good forecast of key macroeconomic variables related to monetary policy and, adequate knowledge about implications of monetary policy actions for relevant target variables. A variety of models are utilized for forecasting and assessing the effects of monetary policy on key macro variables. Among these models, multi-equation or simultaneous equation models are preferred over single equation (regression) models because the former can handle simultaneity issue due to cause and effect of monetary policy actions. Apart from dealing with simultaneity issue, multi-equation models allow broader and more comprehensive analysis of dynamic relationships existing in the economic variables. These models can be categorized among three broad classes: Vector Autoregressive (VAR) models, Macro-econometric models and Dynamic Stochastic General Equilibrium (DSGE) models.

VAR and Macro-econometric models have two major drawbacks: absence of micro foundations and Lucas-critique (Lucas 1976). Efforts to overcome these issues have culminated into a permanent place of dynamic stochastic general equilibrium models (DSGE) in toolkits of economists at many developed economies' central banks. These models have at least four key features which make them particularly attractive for policy analysis. First, DSGE models can allow holistic analysis due to their general equilibrium nature. Second, these models can better model expectations due to micro-founded structure. Third, estimated DSGE models can highlight the uncertainty related to forecasts. Finally, these models are capable of modeling different policy regimes by incorporating policy rules such Taylor type interest rate rule and/or McCallum type money-feedback rules. On the basis of these properties, the usefulness of DSGE models for policy analysis is well established in literature (Clarida et al. 1999).

Despite several academic attractions, initial versions of DSGE models were less useful for real time policy analysis owing to their poor data fitting capabilities. Although rich in terms of micro-founded economic structure yet, their ability to replicate historical macro data or forecast future developments was limited when compared against empirical models such as VARs (Pagan 2003). Main reason behind poor fit was incomplete economic modeling of real and nominal frictions in earlier versions of DSGE models. This issue was addressed by Christiano et al. (2005) through incorporation of real and nominal frictions in the model (Adolfson et al. 2007). These frictions included price rigidity, wage rigidity, indexation, investment adjustment costs, consumption habit formation and variable capacity utilization. Incorporation of these frictions give birth to a "new-generation" ${ }^{1}$ of DSGE models which is at par with VAR models in terms of forecasting performance (Smets and Wouters 2007).

Macro policy makers are especially concerned with the forecasting capabilities of models used for policy analysis. Therefore, in literature, policy usefulness of the new generation of DSGE models is investigated on the basis of forecasting performance of these models. To this end, out-of-sample forecasts of key

1 This term has been used by Adolfson et al. (2005). 
macro variables from DSGE and other competing empirical approaches ${ }^{2}$ are generated and compared (Adolfson et al. (2007), Smets and Wouters (2007), Christoffel et al. (2008), Edge and Gurkaynak (2010), and Edge et al. (2010)). In this regard, most of the studies have been conducted in context of USA or Euro Zone. These studies find that forecasting performance of medium-scale estimated DSGE models is fairly comparable with BVAR and other judgmental forecasts.

Against this backdrop, it is not a surprise that many central banks across the globe use DSGE models for macro policy analysis. Tovar (2009) mentions a few such countries including many developed and emerging economies such as Canada, England, Chile, Peru, USA, Norway, and Sweden. Use of such models by institutes like European Union and International Monetary Fund has also been documented in Christoffel et al. (2008), Berg et al. (2006a) and Berg et al. (2006b), respectively. There have been some serious efforts to use these models for monetary policy analysis in Pakistan as well. In this direction, seminal work was attempted by Haider and Khan (2008) and then subsequent studies, Choudhri and Malik (2012), Ahmad et al. (2012), Haider et al. (2012), Ahmad and Pasha (2015), Ahmad et al. (2016), and Rehman et al. (2017) are notable examples. However, none of these studies analyzes usefulness of micro-founded and estimated DSGE models on the basis of comparison of forecast performance.

This study aims to complement the literature by: first, Bayesian estimation of a small open economy DSGE model with wage and price rigidities, investment adjustment costs, variable capacity utilization and incomplete asset markets. Second, forecasting performance of estimated DSGE model is evaluated through comparison of recursive forecasts for inflation, GDP growth, exchange rate and interest rate. In this phase, forecasts from different versions of VAR (VARX, BVAR and BVARX) models have been compared with DSGE model's forecasts for evaluation purpose. In order to estimate these models, quarterly data of inflation, GDP growth rate, exchange rate and interest rate are used. US inflation, GDP growth and interest rate are considered as proxies for corresponding worldwide indicators that have exogenous impact on Pakistan economy.

The key motivation of this analysis is to answer the question that which model, among an estimated DSGE and different versions of VAR (VARX, BVAR and BVARX) models produces the best out-of-sample forecast for inflation, output growth rate, exchange rate and interest rate in Pakistan. The study analyzes forecasting strengths and weaknesses of estimated DSGE and VAR models in context of Pakistan.

Rest of the paper is organized as: section 2 discusses the relevant literature, section 3 briefly describes the DSGE and VAR models used in the paper, section 4 presents details about data and estimation of models. Section 5 describes forecasting procedure and comparison of different models' forecasts and section 6 concludes. Appendix provides log-linearized version of model along with empirical results.

\footnotetext{
${ }^{2}$ A number of empirical benchmark models such as random walk, VAR, Bayesian VAR and other judgmental forecasts are used for comparison purposes.
} 


\section{Literature review}

The literature exploring usefulness of DSGE models in context of monetary policy analysis typically compares the out-of-sample forecasting power of the DSGE models against different competing models such as BVAR and other judgmental forecasts. For instance, Smets and Wouters (2007) compare the out-of-sample forecasting performance of a DSGE model with BVAR model for USA. For estimation of the model, they use quarterly data (1966:1-2004:4) of seven time series. Another similar study, Edge et al. (2010) evaluates forecast performance of Federal Reserve Board's Estimated DSGE model using real time data set of USA. Edge and Gurkaynak (2010) compare out-of-sample forecasts obtained from Smets and Wouters (2007) model with judgmental forecasts provided by Federal Reserve Staff in Greenbook and BVAR models. Important contribution of this study is evaluation of forecasting performance of Smets and Wouters (2007) model using real time data set. Christoffel et al. (2008) present microfounded derivation, Bayesian estimation and, empirical evaluation of New Area Wide Model (NAWM). It was developed for forecasting and policy analysis at European Central Bank on regular basis. All of the above mentioned studies find that forecasting performance of medium scale estimated DSGE models was fairly comparable with BVAR and other judgmental forecasts.

Considering the merits of DSGE models, there have been a few serious efforts in the areas of construction, calibration and estimation of these models in Pakistan. Most of these studies use DSGE models in order to analyze certain research questions rather than forecasting or real time monetary policy analysis.

For instance, objective of Haider and Khan (2008) is Bayesian estimation and interpretation of structural parameters of DSGE model. They develop a small open economy DSGE model for Pakistan. On the basis of estimated coefficients, they conclude that monetary policy strongly responds to fluctuations in inflation and consumption is almost indifferent to fluctuations in prices.

Choudhri and Malik (2012) use DSGE framework in order to analyze consequences of fiscal dominance for conduct of monetary policy in Pakistan. Their results show that application of Taylor-type interest rate rule in presence of fiscal dominance causes increase in volatility of inflation. Ahmad et al. (2012) use DSGE framework to study the consequences of of informal sector for conduct of monetary and fiscal policies. The results of their study indicate crowing out as a result of fiscal policy and lower response of monetary policy owing to presence of informal sector in production and labor markets. In another study, Choudhary and Pasha (2013) use RBC models to analyze FDI shocks.

Ahmad et al. (2016) utilize DSGE framework in order to analyze the role of nominal factors in business cycles of Pakistan. They try to explain the business cycle fluctuations of key macro variables using DSGE models comprising monetary policy and price rigidity to conclude that DSGE models with explicit incorporation of money have better data matching capabilities as compared to interest rate based monetary policy models. Rehman et al. (2017) use a small open economy DSGE model to study the impact of workers' remittances on key macro variables in Pakistan. The authors show that workers' remittances boost growth through increased consumption and imported investment.

Business Review: (2019) 14(1):28-52 


\section{S. Ahmad, A. Haider}

To summarize, most of the studies using DSGE models in Pakistan seek to utilize these models in order to analyze specific macroeconomic research issues related to fiscal dominance, informal sector, role of FDI shocks, role of nominal factors in business cycles and workers' remittances. These issues are indeed relevant and DSGE models can be used to analyze them. However, as explained in introduction section, modern DSGE models are particularly suitable for real time forecasting and policy analysis on regular basis. This involves use of DSGE models to obtain conditional and unconditional forecasts, counter factual scenario analysis and gap analysis etc.

In this regard, Ahmad and Pasha (2015) show that use of DSGE models for forecasting and policy analysis can improve policy making process by increasing accuracy of forecasts for Pakistan economy. The authors operationalize a version of Forecasting and Policy Analysis System (FPAS) for monetary policy analysis at SBP and document that in case of moderate inflation, forecasting performance of FPAS is better than a suite of econometric models. However, there are three main issues that need to be addressed. First, although a DSGE, yet FPAS is a reduced form model and abstracts from explicit micro foundations. Second, FPAS is a calibrated DSGE model. Bayesian MLE can potentially further increase forecasting power of the model. Finally, the model has been evaluated based on forecasting performance of single variable i.e. inflation only. The current paper aims to improve on the above mentioned issues by Bayesian estimation of a fully micro-founded DSGE model and then analysis of forecasting performance of this model on the basis of a set of key macro variables including inflation, output growth rate, exchange rate and interest rate in context of Pakistan economy.

\section{Description of models}

This section provides brief overview of DSGE and VAR models.

\subsection{DSGE Model}

We estimate a variant of Adolfson et al. (2007) (henceforth, ALLV2007) model for Pakistan economy. There are several reasons for choosing ALLV2007 model as a basic framework. First, the model has enough quantum of nominal and real frictions to render it a useful model for monetary policy analysis. Second, the model is a small open economy model and extensive modeling of external sector makes this model particularly suitable for analyzing exchange rate fluctuations. Third, the framework presented in the model serves as the cornerstone of many central banks' DSGE models and allows comparison of results across similar economies. ${ }^{3}$ Fourth, the model allows relatively easy customization. Finally, apart from monetary policy, the model incorporates different types of taxes and can be used quite efficiently for fiscal policy analysis as well.

\footnotetext{
3 The model is included in Macro Models Base; an initiative to facilitate comparison of DSGE models by (Wieland et al. 2012)
} 
Before estimating the model using Pakistan economy data, we do a few changes in the model. Objective of these changes is to simplify the model. First change is related to VAR based modeling of fiscal and external blocs. For these blocs, we replace VAR based models by simple first order autoregressive processes. Second change is related to time varying mark-ups. Instead of variable mark-ups, we use constant mark-ups. Third, we exclude interest rate reaction to exchange rate fluctuations from Taylor type interest rate rule. Finally, we remove the friction between hours worked and employment due to lack of employment hours and wage rate data in case of Pakistan.

The model mainly captures the behavior of five sectors: firms, households, fiscal authority, monetary authority and external sector. Firms are divided in three broad classes: domestic firms, importing firms and exporting firms. Importing firms are further classified as consumption goods importing firms and investment goods importing firms. All types of firms face Calvo (1983) type time dependent price revision constraints. Households derive utility from consumption, leisure and cash holding. Households' consumption and investment contain domestic as well as foreign components.

Households' wages are subject to Calvo-type wage-revision constraints on the lines of (Erceg et al. 2000). Households' preferences are subject to habit persistence and they face real frictions like investment adjustment costs and variable capacity utilization. These modeling features are necessary to capture important dynamic effects of monetary policy actions on inflation, output, consumption and investment (Christiano et al. 2005).

Fiscal authority runs a balanced budget. Expenditure side includes fiscal spending and transfer payments. Revenues are gathered from different types of taxes such as labor income tax, capital income tax, payroll tax, consumption tax and, seigniorage. To model different tax rates, we use either autoregressive processes or constant average tax rates. Fiscal spending has also been modeled as an autoregressive process.

Central bank adjusts nominal interest according to Taylor type interest rate rule. Taylor rule incorporates interest rate smoothing, inflation and output gap fluctuations. Dynamic behavior of foreign variables has also been modeled through autoregressive process for inflation, output and interest rate. Key linearized equations of the equilibrium model are presented in Appendix A. Notations of the model variables and parameters are similar to Adolfson et al. (2005).

\subsection{VARX Model}

Sims (1980) presented the idea of Vector Autoregressive (VAR) models. In these models, all macro variables are considered endogenous. This property can be regarded as the greatest strength of VAR models. By including all variables, each variable gets a fair chance to show its impact in forecast. Thus, the forecast coming from VAR models is based on a richer set of dynamic relationships when compared against tightly theorized macroeconometric or DSGE models.

Business Review: (2019) 14(1):28-52 


\section{S. Ahmad, A. Haider}

In order to stay consistent with small open economy situation, we introduce foreign economy variables as exogenous variables in VAR model. This implies that foreign variables affect domestic variables however due to atomistic size of domestic economy in comparison to world economy, converse is not true. A VAR model with exogenous variables is called VARX model (Ouliaris et al. 2016).

Since our interest lies in forecasting rather than structural analysis, therefore we estimate reduced form VAR models. A reduced form VAR is a multiple equation system in which an economic variable depends upon its own and, other variables' lagged values. General form of reduced form VAR model with exogenous variables (i.e. VARX) is given by:

$$
y_{t}=\sum_{l=1}^{L} A_{l} y_{t-l}+\sum_{m=0}^{M} B_{m} x_{t-m}+\varepsilon_{t}
$$

where $y_{t}$ is a vector of endogenous variables included in estimation with $L$ lags and $x_{t}$ is a vector of exogenous variables included in estimation with $M$ lags. Elements of error term vector $\varepsilon_{t}$ are uncorrelated with their lag values and other explanatory variables. In our case, $y_{t}=\left[\Delta G D P_{t} \pi_{t} \Delta S_{t} i_{t}\right]^{\prime}$ is vector of endogenous variables. Foreign variables are included in vector $x_{t}=$ $\left[\alpha \Delta G D P_{t}^{U S A} \pi_{t}^{U S A} i_{t}^{U S A}\right]^{\prime}$. Constant $\alpha$ represents inclusion of intercept.

VAR models are mainly criticized for over parameterization problem. Including a large number of variables and time lags involves estimation of a huge number of parameters. ${ }^{4}$ Time series data may not be able to fulfill requirements of VAR models for two reasons. First, long time series simply might not be available. Second, time series data could be plagued by structural breaks or regime shifts.

\subsection{Bayesian VARX Model}

Scarcity of time series data may lead to poor estimation of VAR model. Apart from the issue of data scarcity, Litterman (1986) highlighted the issue of relevance of old macro time series data in context of forecasting. He pointed out that due to changing nature of shocks, business cycle and other factors, distant past time series data may be quite irrelevant for the purpose of forecasting. Overparameterization is still another issue that deteriorates forecast performance of VAR models. Parsimonious models are considered better than over-fitted models in terms of their forecasting performance (Ouliaris et al. 2016).

These issues can be tackled by using Bayesian VAR approach proposed by Litterman (1986). By using appropriate priors, a researcher can control the importance of estimated parameters in the VAR model and over-fitting problem can be avoided. Good forecasting performance of BVAR models has been widely documented in literature. Bloor and Matheson (2011) show that BVAR model outperforms other univariate and multivariate models' forecasts. Todd (1984) and Doan et al. (1986) also observed good forecasting potential of BVAR models.

\footnotetext{
4 If we have $n$ variables and want to estimate a VAR model with $L$ lags, then we need to estimate $n(1+n L)$ number of coefficients. For instance, for a 3 variable and 4 lags VAR model, we need to estimate 39 coefficients
} 
Prior information is incorporated in the form of hyper-parameters. Since number of hyper-parameters is considerably less than actual parameters in VAR model, therefore this approach effectively reduces dimension of VAR model parameters. We know that OLS parameters are estimated through following estimator:

$$
\hat{\beta}=\left(x^{\prime} x\right)^{-1} x^{\prime} y \quad \sim N(\underline{b}, \underline{V})
$$

where $\underline{b}$ and $\underline{\mathrm{V}}$ respectively represent prior mean and variance-covariance matrices of $\hat{\beta}$. Prior values are assumed to coincide with population statistics. In case of normally distributed conjugate priors, posterior will also be normal. In such a case, mean (and mode) of posterior distribution is a matrix weighted average of OLS estimates and priors (or hyper-parameters).

$$
\overline{\hat{\beta}}=\left[\underline{\mathrm{V}}^{-1}+\Sigma_{e}^{-1} \otimes\left(x^{\prime} x\right)\right]^{-1}\left[\underline{\mathrm{V}}^{-1} \underline{\mathrm{b}}+\left(\Sigma_{e}^{-1} \otimes x^{\prime}\right) y\right]
$$

where $\overline{\hat{\beta}}$ represent posterior mean (=mode in case of normal posterior) estimate of coefficients.

We use Minnesota priors which are summarized by four hyper-parameters $\mu_{1}, \lambda_{1}, \lambda_{2}$ and $\lambda_{3}$. $\mu_{1}$ sets the magnitude of first order autocorrelation. For highly persistent but stationary data, $\mu_{1}$ is set close to 1 . In case of differenced data, value of $\mu_{1}$ should be close to $0 . \lambda_{1}$ captures uncertainty regarding mean values of first order persistence parameters. Smaller value of $\lambda_{1}$ indicates greater amount of confidence about prior mean value and vice versa. For very large values of $\lambda_{1}$, posterior estimates converge to OLS estimates (Ouliaris et al. 2016). $\lambda_{2} \in(0,1)$ measures the impact of cross variables. If current values of endogenous variables are strongly affected by lags of other variables, then $\lambda_{2}$ should be close to 1 ; otherwise $0 . \lambda_{3}$ measures lag decay rate. Higher the magnitude of $\lambda_{3}$, higher will be lag decay rate implying lower importance of higher lags.

\section{Data and estimation of models}

\subsection{Data}

We use quarterly data (1980Q4-2017Q2) of real GDP, CPI, USD/PKR exchange rate and Call Money Rate. Since actual quarterly data of GDP are not available in Pakistan, therefore we have to use approximated GDP data from Hanif et al. (2013) where this series is available till 2012Q2. For 2012Q2-2017Q2, we interpolate annual real GDP series using most recent seasonal proportions from Hanif et al. (2013). For foreign variables, we use USA GDP, CPI and 3-months T-Bill rate. We need quarterly population series in order to get per capita real GDP. Annual series of Pakistan and US population have been taken from UN Population Statistics and quarterly series are obtained through cubic interpolation. Details about data and variables are summarized in Table B.1 (please see Appendix). 


\subsection{Bayesian estimation of DSGE Model}

There are two broad ways to parameterize the DSGE models: calibration and estimation. Parameters of initial general equilibrium models ${ }^{5}$ were assigned numeric values through calibration method. Calibration involves using information from micro economic studies, long term data analysis to find great ratios and, using information from other studies to measure the values of model parameters (Heijdra and Ploeg 2002). However, this method has two main limitations. First, calibration method does not provide systematic way to assess models' capability to match actual data. Second, this method does not provide a coherent method for comparison of different models. Maximum likelihood estimation (MLE) is another method to parameterize the DSGE models. However, MLE based parameterization has also some issues. First, likelihood function can be extremely complex and non-linear function of parameters. Second, likelihood function can be very flat and optimization could be difficult. Finally, likelihood function can provide parameter estimates which are against economic logic.

To effectively overcome these issues, modern studies using DSGE models generally use a combination of calibration and Bayesian maximum likelihood estimation methods to parameterize models (Smets and Wouters (2003), Smets and Wouters (2007) and Adolfson et al. (2007)). In this approach, one set of parameters is calibrated and rest of the parameters are estimated through Bayesian maximum likelihood method. In cases where parameter values could be found with substantial degree of confidence from literature or simple data analysis (ratios, proportions and shares etc.), we use calibration approach.

In cases where we do not have substantial evidence from literature, we use Bayesian maximum likelihood estimation approach to find numeric value of the parameter. This estimation technique has certain advantages over onlycalibration and other estimation methods. First, Bayesian estimation method uses prior information in the form of prior distributions for model parameters. This adds to information set and enables estimation of parameters in economically meaningful range. Second, Bayesian estimation allows statistical evaluation of model fit and comparison across different models. Finally, Bayesian estimation is the middle path between fixed parameters (i.e. calibration) and completely data driven parameter estimates (i.e. Maximum Likelihood Estimation). ${ }^{6}$

For Bayesian estimation of model, number of observed variables and measurement errors should be equal to number of shocks used in the model. Since we use 7 data series, therefore we introduce 7 shocks in the model. We include 4 domestic and 3 foreign shocks. Domestic shocks include technology shock, monetary policy shock, fiscal policy shock and foreign exchange risk premium shock. Foreign shocks include foreign demand, foreign inflation and world interest rate. Model variables and observed data are linked through measurement equations. As we have 7 variables and shocks, naturally, we have 7 measurement equations. State space representation of rational expectations solution of DSGE model is given as:

\footnotetext{
5 see for instance, Cooley and Hansen (1989).

6 It is interesting to note that Bayesian estimation under uniform distribution of parameters is equivalent to maximum likelihood estimation of the model.
} 


$$
X_{t}=R X_{t-1}+S \epsilon_{t}
$$

where $X_{t}$ is a vector of state variables of the model. Since all of the variables are not observable, therefore measurement equation relates model variables with actual data.

$$
X_{t}^{o b s}=\Gamma+V X_{t}+e_{t}
$$

where $X_{t}^{\text {obs }}$ is vector of observable variables, $\Gamma$ is vector of constants and $V$ is the matrix selecting and linking state and observable variables. In our case,

$$
\begin{gathered}
X_{t}^{\text {obs }}=\left[\widehat{Y}_{t} \pi_{t} \Delta S_{t} R_{t} \widehat{Y}_{t}^{U S A} \pi_{t}^{U S A} R_{t}^{U S A}\right]^{\prime} \\
X_{t}^{\text {obs }}=\left[\begin{array}{c}
\widehat{y}_{t}^{o b s} \\
\pi_{t}^{\text {obs }} \\
\Delta S_{t}^{\text {obs }} \\
R_{t}^{\text {obs }} \\
\widehat{Y}_{t}^{U S A, o b s} \\
\pi_{t}^{U S A, o b s} \\
R_{t}^{U S A, o b s}
\end{array}\right]=\left[\begin{array}{c}
100\left(\mu_{z}^{4}-1\right)+400\left(\widehat{y}_{t}-\widehat{y}_{t-1}\right) \\
100\left(\pi^{4}-1\right)+400 \pi_{t} \\
\overline{\Delta S}+100 \Delta S_{t} \\
100\left(\pi^{4}-1\right)+400 \hat{R}_{t} \\
100\left(\mu_{z}^{* 4}-1\right)+400\left(\widehat{y}_{t}^{U S A}-\widehat{y}_{t-1}^{U S A}\right) \\
100\left(\pi^{U S A^{4}}-1\right)+400 \pi_{t}^{U S A} \\
100\left(\pi^{U S A^{4}}-1\right)+400 \hat{R}_{t}^{U S A}
\end{array}\right]=\Gamma+V X_{t}
\end{gathered}
$$

\subsubsection{Calibration of parameters}

While calibrating parameters, we mainly rely on literature and choose appropriate values for quarterly frequency of data. Subjective discount factor, $\beta$ is calibrated to be 0.99 ; implying annual real risk-free interest rate of $4 \%$. Value of quarterly depreciation rate, $\delta$ is fixed at 0.0164 following (Ahmad et al. 2016). Share of capital in production function, $\alpha$ is calibrated to be 0.5 following (Ahmad et al. 2012). Scaling parameter $A_{L}=5.2$ provides disutility of labour supply. This parameter has been fixed in such a way that steady state proportion of representative household worked hours is 0.28 (Ahmad et al. 2016). Wage markup $\lambda_{W}$ measures the extent of monopolistic competition in labour market. We choose a value of 1.05 for this parameter. Markup coefficients for domestic producers, consumption goods importers and investment goods importers $\left(\lambda_{d}, \lambda_{m c}\right.$ and $\left.\lambda_{m i}\right)$ are assumed equal and calibrated to be 1.2. Shares of imported goods in investment and consumption goods $\omega_{I}$ and $\omega_{C}$ are calibrated to be 0.30 . Ratio of fiscal spending to GDP $\bar{g}$, has been fixed at 0.20 by taking arithmetic mean of the variable over the sample period.

Considering relatively low tax-GDP ratio in Pakistan, steady state income tax rate $\tau_{Y}$ has been fixed at 0.10 (or 10\%). Steady state value added tax rate $\tau_{C}$, is calibrated to be $0.17(17 \%)$ in line with the sales tax rate in Pakistan. Elasticity of substitution between domestic and foreign consumption goods, $\eta_{C}$ and the corresponding coefficient for investment goods $\eta_{I}$ are calibrated to be on lower side. For both parameters, we choose a value of 0.8 . A lower value of elasticity can be interpreted as lower degree of substitutability between domestic and foreign goods. Potential presence of cost channel of monetary policy in case of Pakistan has been noted in literature (Javid and Munir 2010). In order to activate this channel in model, we assume that proportion of firms needing external financing to pay their wage bill $(\nu)$ is equal to $0.2(20 \%)$.

Business Review: (2019) 14(1):28-52 
Table 1: Calibrated parameters

\begin{tabular}{|c|c|c|c|}
\hline Sr. No. & Symbol & Parameter & Value \\
\hline 1 & $\beta$ & Discount factor & 0.99 \\
\hline 2 & $\eta_{c}$ & Elasticity of subs. bw. domestic and foreign consumption goods & 0.8 \\
\hline 3 & $\eta_{i}$ & Elasticity of subs. bw. domestic and foreign investment goods & 0.8 \\
\hline 4 & $\eta_{f}$ & Elasticity of subs. bw. different countries goods & 2 \\
\hline 5 & $A_{q}$ & Weight of cash holding in utility function & 0.88 \\
\hline 6 & $A_{L}$ & Weight of labour supply in utility function & 5.2 \\
\hline 7 & $\omega_{i}$ & Share of foreign goods in total investment & 0.3 \\
\hline 8 & $\omega_{c}$ & Share of foreign goods in total consumption & 0.3 \\
\hline 9 & $\lambda^{w}$ & Steady state mark-up for labour supply & 1.05 \\
\hline 10 & $\lambda^{d}$ & Mark-up for domestic goods & 1.2 \\
\hline 11 & $\lambda^{m, c}$ & Mark-up for imported consumer goods & 1.2 \\
\hline 12 & $\lambda^{m, i}$ & Mark-up for imported investment goods & 1.2 \\
\hline 13 & $\xi_{w}$ & Calvo price stickiness index for wages & 0.7 \\
\hline 14 & $\xi_{d}$ & Calvo price stickiness index for domestic goods & 0.25 \\
\hline 15 & $\xi_{m, c}$ & Calvo coefficient for imported consumption goods & 0.25 \\
\hline 16 & $\xi_{m, i}$ & Calvo coefficient for imported investment goods & 0.25 \\
\hline 17 & $\xi_{x}$ & Calvo coefficient for exported goods & 0.7 \\
\hline 18 & $\kappa_{w}$ & Wage indexation coefficient & 0.5 \\
\hline 19 & $\kappa_{d}$ & Price indexation for domestic goods & 0.3 \\
\hline 20 & $\kappa_{m, c}$ & Price indexation for imported consumer goods & 0.3 \\
\hline 21 & $\kappa_{m, i}$ & Price indexation for investment goods & 0.3 \\
\hline 22 & $\bar{g}$ & Steady state fiscal spending to GDP ratio & 0.2 \\
\hline 23 & $\mu$ & Steady state money growth rate & 1.025 \\
\hline 24 & $\tau_{y}$ & Steady state labor income tax & 0.1 \\
\hline 25 & $\tau_{c}^{9}$ & Steady state value added tax & 0.17 \\
\hline 26 & $\tau_{w}$ & Steady state payroll tax & 0.05 \\
\hline 27 & $\tau_{k}$ & Steady state capital income tax & 0.2 \\
\hline 28 & $\alpha$ & Capital share in production & 0.5 \\
\hline 29 & $\delta$ & Depreciation rate & 0.0164 \\
\hline 30 & $\mu_{z}$ & Steady state tech. growth rate & 1.006 \\
\hline 31 & $\bar{\pi}$ & Steady state inflation & 1.019 \\
\hline 32 & $\nu$ & Share of firms need working finance & 0.2 \\
\hline 33 & $\tilde{S}$ & Investment adjustment costs curvature & 12.67 \\
\hline 34 & $\sigma_{a}$ & Capital utilization costs coefficient & 10.6 \\
\hline 35 & $\sigma_{q}$ & Inverse elasticity of cash holding demand & 10.62 \\
\hline
\end{tabular}

Calvo wage stickiness index $\xi_{W}=0.70$ is taken from (Ahmed et al. 2014). This implies that in a given quarter, $70 \%$ of households are not able to reset their wage rate according to their preferences. Calvo price stickiness coefficients for domestically produced goods $\xi_{d}=0.25$, is taken from (Choudhary et al. 2016). Local currency pricing implies that Calvo price stickiness indices for imported consumption and imported investment goods $\left(\xi_{m c}\right.$ and $\left.\xi_{m i}\right)$ represent behavior of domestic importing firms rather than their foreign producers. Owing to lack of any direct microeconomic evidence related to these parameters, we assume that $\xi_{m c}$ and $\xi_{m i}$ are equal to $\xi_{d}$. Using same reasoning, we assume that Calvo price stickiness index for exported goods $\xi_{X}$ is equal to foreign counterpart of $\xi_{d}$. Using information from Choudhary et al. (2016), we fix value of $\xi_{X}$ at 0.70 .

Wage indexation parameter $\kappa_{W}$ is calibrated to be 0.50 . Indexation coefficients related to domestic prices, imported consumption and investment goods and, exported goods are assumed equal $\left(\kappa_{d}, \kappa_{m c}, \kappa_{m i}, \kappa_{X}=0.30\right)$. All calibrated parameters are summarized in table 1 . 


\subsubsection{Priors for Bayesian estimation process}

We use Beta distribution for parameters whose values lie between 0 and 1 and Gamma distribution in cases where economic rationale calls for strict positivity of a coefficient. Inverse Gamma distribution has been assumed for standard deviations of shock processes.

Table 2: Estimated structural parameters

\begin{tabular}{|c|c|c|c|c|c|c|c|c|}
\hline \multirow[b]{2}{*}{ Sr. No. } & \multirow[b]{2}{*}{ Symbol } & \multirow[b]{2}{*}{ Parameter } & \multirow[b]{2}{*}{ Prior Dist. } & \multicolumn{2}{|l|}{ | Prior } & \multicolumn{3}{|c|}{ Posterior } \\
\hline & & & & | Mean | & SD & Mode & Mean & SD \\
\hline 1 & $b$ & Habit formation in consumption & Beta & 0.6 & 0.1 & 0.557 & 0.576 & 0.151 \\
\hline 2 & $\sigma_{L}$ & Inverse elasticity of labour supply & Gamma & 0.8 & 0.25 & 0.722 & 0.807 & 0.237 \\
\hline 3 & $\phi_{a}$ & Sensitivity of exchange rate changes to FX risk premium & Gamma & 0.2 & 0.065 & 0.005 & 0.006 & 0.002 \\
\hline 4 & $\rho_{R}$ & Interest rate smoothing in interest rate rule & Beta & 0.9 & 0.15 & 0.88 & 0.882 & 0.033 \\
\hline 5 & $\rho_{\pi}$ & Monetary policy response to inflation & Gamma & 3.8 & 1.2 & 2.561 & 2.793 & 0.751 \\
\hline 6 & $\rho_{y}$ & Monetary policy response to output gap & Gamma & 1.03 & 0.3 & 0.199 & 0.241 & 0.078 \\
\hline
\end{tabular}

Prior mean for habit formation coefficient $b$ is assumed to be 0.60 . This is consistent with a substantial amount of inertia in consumption; generally observed in data. Adolfson et al. (2007) report posterior mean of 0.69 for habit formation. Prior mean for inverse elasticity of labor supply $\sigma_{L}$, is fixed at 0.8 . Mean for sensitivity of exchange rate changes to FX risk premium has been set at 0.20 based on correlation between exchange rate change and a risk premium calculated from UIP condition. Priors for coefficients of Taylor-type interest rate reaction function are taken from (Ahmad et al. 2016). Prior means for degree of interest rate smoothing $\rho_{R}$, response to inflation $\rho_{\pi}$ and response to output gap $\rho_{y}$ are set at $0.90,3.80$ and 1.03 , respectively (see, table 2 and 3 ).

Table 3: Estimated shock parameters

\begin{tabular}{|c|c|c|c|c|c|c|c|c|}
\hline \multirow[b]{2}{*}{ Sr. No. } & \multirow[b]{2}{*}{ Symbol } & \multirow[b]{2}{*}{ Parameter } & \multirow[b]{2}{*}{ Prior Dist. } & \multicolumn{2}{|l|}{ Prior } & \multicolumn{3}{|c|}{ Posterior } \\
\hline & & & & Mean & SD & Mode & Mean & $\mathrm{SD}$ \\
\hline 1 & $\rho_{\varepsilon}$ & Persistence of transitory tech. shock & Beta & 0.5 & 0.1 & 0.885 & 0.8639 & 0.027 \\
\hline 2 & $\rho_{g}$ & Persistence of fiscal spending shock & Beta & 0.5 & 0.1 & 0.937 & 0.9351 & 0.011 \\
\hline 3 & $\rho_{\tilde{\phi}}$ & Persistence of FX risk prem. shock & Beta & 0.5 & 0.1 & 0.969 & 0.967 & 0.011 \\
\hline 4 & $\rho_{\phi^{*}}$ & Persistence of foreign inflation shock & Beta & 0.5 & 0.1 & 0.547 & 0.547 & 0.054 \\
\hline 5 & $\rho_{y^{*}}$ & Persistence of foreign demand shock & Beta & 0.5 & 0.1 & 0.961 & 0.9603 & 0.009 \\
\hline 6 & $\rho_{R^{*}}$ & Persistence of foreign interest rate shock & Beta & 0.5 & 0.1 & 0.948 & 0.9469 & 0.012 \\
\hline 7 & $\sigma_{\varepsilon}$ & SD of transitory tech. shock & Inv. Gamma & 0.01 & 0.01 & 0.014 & 0.0139 & 0.001 \\
\hline 8 & $\sigma_{q}$ & SD of fiscal spending shock & Inv. Gamma & 0.01 & 0.01 & 0.118 & 0.117 & 0.0107 \\
\hline 9 & $\sigma_{R}$ & SD of monetary policy shock & Inv. Gamma & 0.01 & 0.01 & 0.005 & 0.0053 & 0.0004 \\
\hline 10 & $\sigma_{\tilde{\phi}}$ & SD of FX risk prem. shock & Inv. Gamma & 0.01 & 0.01 & 0.005 & 0.0053 & 0.001 \\
\hline 11 & $\sigma_{\pi^{*}}^{\phi}$ & SD of foreign inflation shock & Inv. Gamma & 0.01 & 0.01 & 0.005 & 0.0048 & 0.0003 \\
\hline 12 & $\sigma_{y^{*}}$ & SD of foreign inflation shock & Inv. Gamma & 0.01 & 0.01 & 0.007 & 0.0072 & 0.0004 \\
\hline 13 & $\sigma_{R^{*}}^{y^{*}}$ & SD of foreign interest rate shock & Inv. Gamma & 0.01 & 0.01 & 0.002 & 0.0018 & 0.0001 \\
\hline
\end{tabular}

\subsection{Estimation of VARX and BVARX models}

We check stationarity of variables included in VAR models using Augmented Dickey Fuller test. Results are presented in table B.2. These results show that all domestic and foreign variables are $\mathrm{I}(0)$ at $5 \%$ level of significance except call money rate. Null hypothesis for presence of unit root in CMR series can

Business Review: (2019) 14(1):28-52 
be rejected at $17 \%$ level of significance ${ }^{7}$. We prefer to use CMR series without differencing or de-trending due to two reasons. First, our primary objective of VAR models is forecasting rather than structural analysis. Second, inverse roots of AR polynomial show that VAR model estimated with level of interest rate is stable.

Choosing appropriate lag length is one of the crucial elements of VAR estimation (Ouliaris et al. 2016). Considering quarterly data frequency and using Akaike information criteria (AIC), we choose 4 lag for estimation of VAR models (Table B.3). ${ }^{8}$ In case of exogenous variables, contemporaneous terms and 1 lag are included.

We try different combinations of Minnesota priors to obtain minimum forecast errors. Our final choice of hyper-parameters; which is presented in Table 4, implies high degree of first-order persistence (in levels), substantial effect of cross variables and low lag decay rate.

Table 4: Hyper-parameters for BVAR Models

\begin{tabular}{rclc}
\hline Sr. No. & Symbol & Hyper-parameter & Prior value \\
\hline 1 & $\mu_{1}$ & Autoregressive Persistence & 0.2 \\
2 & $\lambda_{1}$ & Tightness of AR coefficients & 0.1 \\
3 & $\lambda_{2}$ & Importance of cross variables & 0.7 \\
4 & $\lambda_{3}$ & Lag decay rate & 0.3 \\
\hline
\end{tabular}

\section{Forecast evaluation and comparison}

We use two basic measures of forecast evaluation for comparison purpose: bias and root mean squared errors. Bias is simply calculated by taking average of forecast errors:

$$
\text { Bias }=\frac{1}{f} \sum_{t=1}^{f}\left(F E_{t}\right)
$$

where $F E_{t}=\hat{X}_{t}-X_{t}$ represents forecast error. A positive value indicates that the forecasting model is; on average, over predicting the forecast variable and vice versa. Commonly used for forecast evaluation purpose, root mean squared error (RMSE) is a measure of distance between forecast and actually realized value of some variable.

\footnotetext{
7 This finding indicates that some of these variables could be co-integrated suggesting VECM. But for short and medium term, VECM forecasts are not likely to differ much from VAR forecasts.

8 Lag length and stability of estimated VAR models were checked at each run of estimation during recursive forecast generation.
} 


$$
R M S E=\sqrt{\frac{1}{f} \sum_{t=1}^{f}\left(F E_{t}\right)^{2}}
$$

A model with lower absolute value of bias and, smaller RMSE is considered better in terms of forecasting.

Since we aim to compare forecasting performance of estimated VAR models with small open economy DSGE model, we estimate VAR models while treating foreign variables as exogenous variables. Exogenous treatment of foreign variables necessitates the provision of forecasts of their values during forecasting of endogenous variables through VAR models. In order to generate forecasts of USA GDP growth, T bill rate and inflation to be used in VAR forecasting exercise, we use similar autoregressive models used in DSGE model. Forecasts of exogenous variables are generated in recursive manner.

$$
\begin{aligned}
\hat{y}_{t}^{*} & =\alpha_{y^{*}}+\rho_{y^{*}} \hat{y}_{t-1}^{*}+\varepsilon_{y^{*}, t} \\
\pi_{t}^{*} & =\alpha_{\pi^{*}}+\rho_{\pi^{*}} \pi_{t-1}^{*}+\varepsilon_{\pi^{*}, t} \\
R_{t}^{*} & =\alpha_{R^{*}}+\rho_{R^{*}} R_{t-1}^{*}+\varepsilon_{R^{*}, t}
\end{aligned}
$$

To carry out expanding window recursive forecast comparison exercise, we initially restrict the model estimation sample to 1980Q4-2008Q4; to pretend as if forecasts are being generated at the end of 2008Q4 and, generate pseudo outof-sample forecast for next 8 quarters i.e. 2009Q1-2010Q4. By comparing the forecasts for 4 key variables of interest (GDP growth, inflation, interest rate and percent change in exchange rate) against their actual values in corresponding quarters, we compute bias and RMSE for this specific 8-quarter forecasting window. We incrementally expand our estimation sample by one observation (1 quarter) to update model parameters and, generate pseudo out-of-sample forecasts for next 8-quarter forecasting window. Our final estimation sample and forecasting window are 1980Q4-2015Q2 and 2015Q3-2017Q2, respectively. Doing so, we get 27 windows of 8 -quarter forecasts for 4 variables.

For each of the four variables, forecast errors can be arranged as an $8 \times 27$ matrix. Utilizing information in forecast-errors matrices, we compute bias and RMSE for different forecast horizons and different time periods. ${ }^{9}$

\subsection{Forecasting performance over different forecast horizons}

Analysis of forecasting performance for different horizons reveals how competing models perform in short run vs. medium run. Bias and RMSEs over different forecast horizons are graphically displayed in Figure 1.

First panel of figure 1 shows forecast performance statistics for annualized GDP growth rate. VAR models appear to be better than DSGE model as they exhibit smaller amount of RMSE for all forecast horizons; except for 8-quarter

\footnotetext{
9 Row-wise computation of bias and RMSE from forecast error matrices yields forecast performance stats over different forecast horizons. Column-wise computation provides bias and RMSE for different time periods.
}

Business Review: (2019) 14(1):28-52 
Fig. 1: Bias and RMSE over different forecast horizons

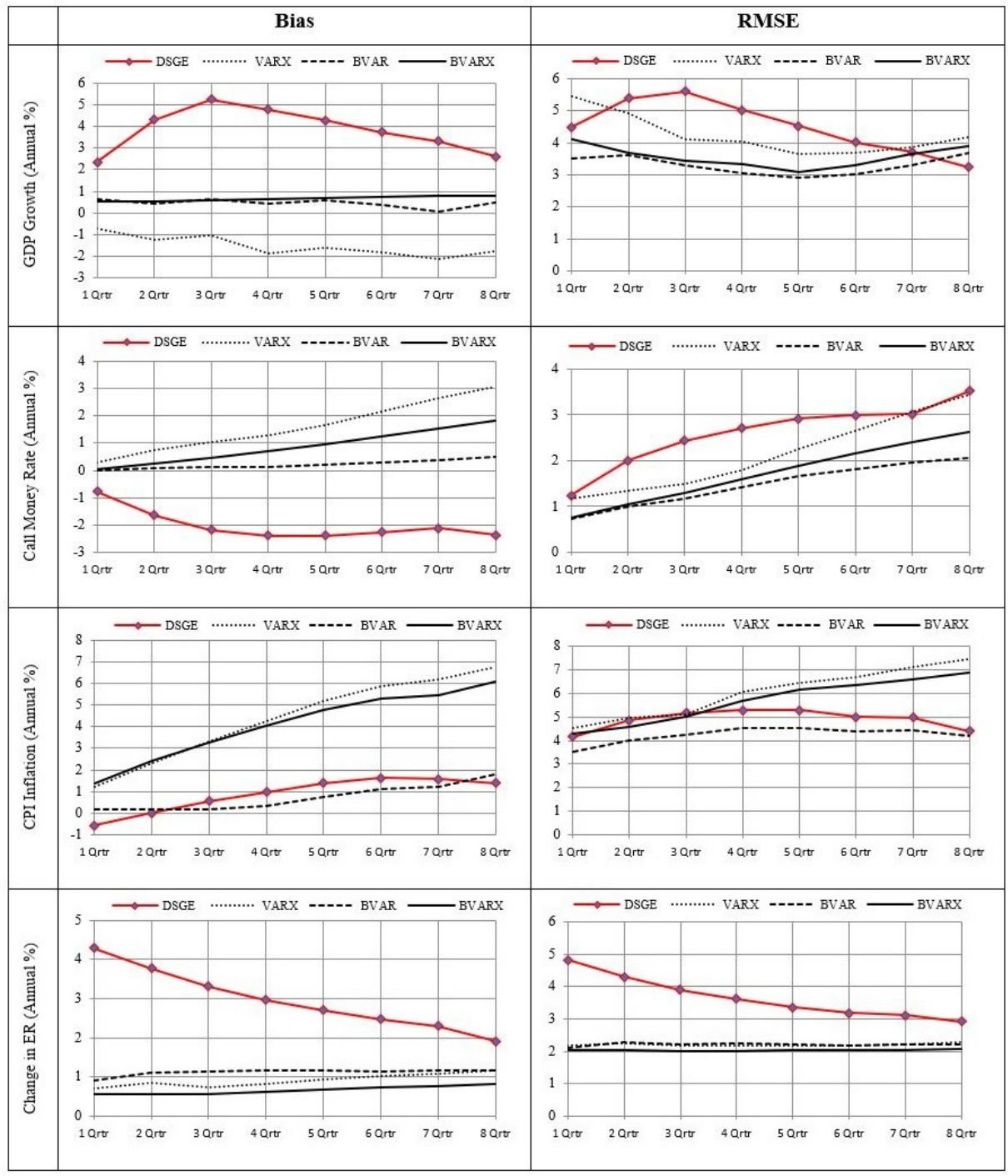

horizon where DSGE model provides minimum RMSE. Among VAR models, BVAR provides lower RMSE and bias than BVARX. This shows that foreign variables included as exogenous variables do not add much information to improve forecast of GDP. RMSE for DSGE model increase for 1-3 quarters but consistently declines after that. This indicates that DSGE model is relatively better at forecasting GDP for medium term horizon rather than short term horizon. DSGE model displays persistently positive bias for all forecast horizons; indicating over prediction. 
Second panel of figure 1 provides information about bias and RMSE in case of annualized call money rate. VAR models provide better forecast for interest rate as RMSE and bias are lower than the same statistics for DSGE model. Among VAR models, BVAR consistently provides minimum amount of RMSE and bias for forecast horizons. Similar to GDP, this shows that foreign variables included in the models are relatively less relevant for forecasting call money rate. For 7-8 quarter horizon, RMSE for DSGE and VARX are almost same.

Third panel of figure 1 provides information regarding bias and RMSE in case of annualized quarter-on-quarter CPI inflation. Here BVAR appears to be the winner as both bias ${ }^{10}$ and RMSE are minimum in case of BVAR model. However, we can see that for longer forecast horizons, DSGE model is second to only BVAR model. Difference between DSGE and BVAR RMSE tends to narrow as forecast horizon increases; indicating towards improvement in DSGE models's inflation forecasting capability over medium term.

Fourth panel of figure 1 provides information about bias and RMSE of percent change in exchange rate (PKR/USD). BVARX appears to be the winner in this case. Bias and RMSE of BVARX are lowest for all forecast horizons. DSGE model forecasts show quite large positive bias as the model does not account for short run FX market interventions designed to avoid domestic currency depreciation. However, as fundamentals tend to prevail in the medium run, both bias and RMSE of DSGE forecast decline.

\subsection{Forecasting performance over different time periods}

Analysis of different models' forecasting performance over different time periods sheds light on these models' forecasting capabilities during different economic environments. Bias and RMSEs over different time period are graphically presented in figure 2.

First panel of figure 2 presents recursively computed bias and RMSE for annualized GDP growth over the period 2009Q1-2017Q2. Bayesian VAR models appear to provide relatively better forecast during our forecast sample (2009Q12017Q2). BVARX provides minimum RMSE during most of the time period. ${ }^{11}$ During 2012Q2-2015Q1, forecasting performance of DSGE model is also very close to VAR models. Apart from RMSE, VAR models provide lower (in absolute terms) amount of bias. For the time period 2009Q1-2014Q4, BVAR model provides minimum bias. In the subsequent period, bias from the three VAR models is very close. For entire forecast sample, DSGE model provides large positive bias. Being a general equilibrium model, DSGE models' forecast errors have structural interpretation. In this case, positive bias implies that our DSGE model persistently indicates towards existence of growth potential in the economy that has not been realized.

Second panel of figure 2 presents bias and RMSE for annualized call money rate. No single model provides minimum RMSE over the sample period. BVAR dominates during most of the samples followed by DSGE during 11Q4-14Q2,

\footnotetext{
10 In absolute value.

11 During 2012Q2-2015Q1, the three VAR models depict similar level of forecast accuracy.
} 
Fig. 2: Bias and RMSE over time

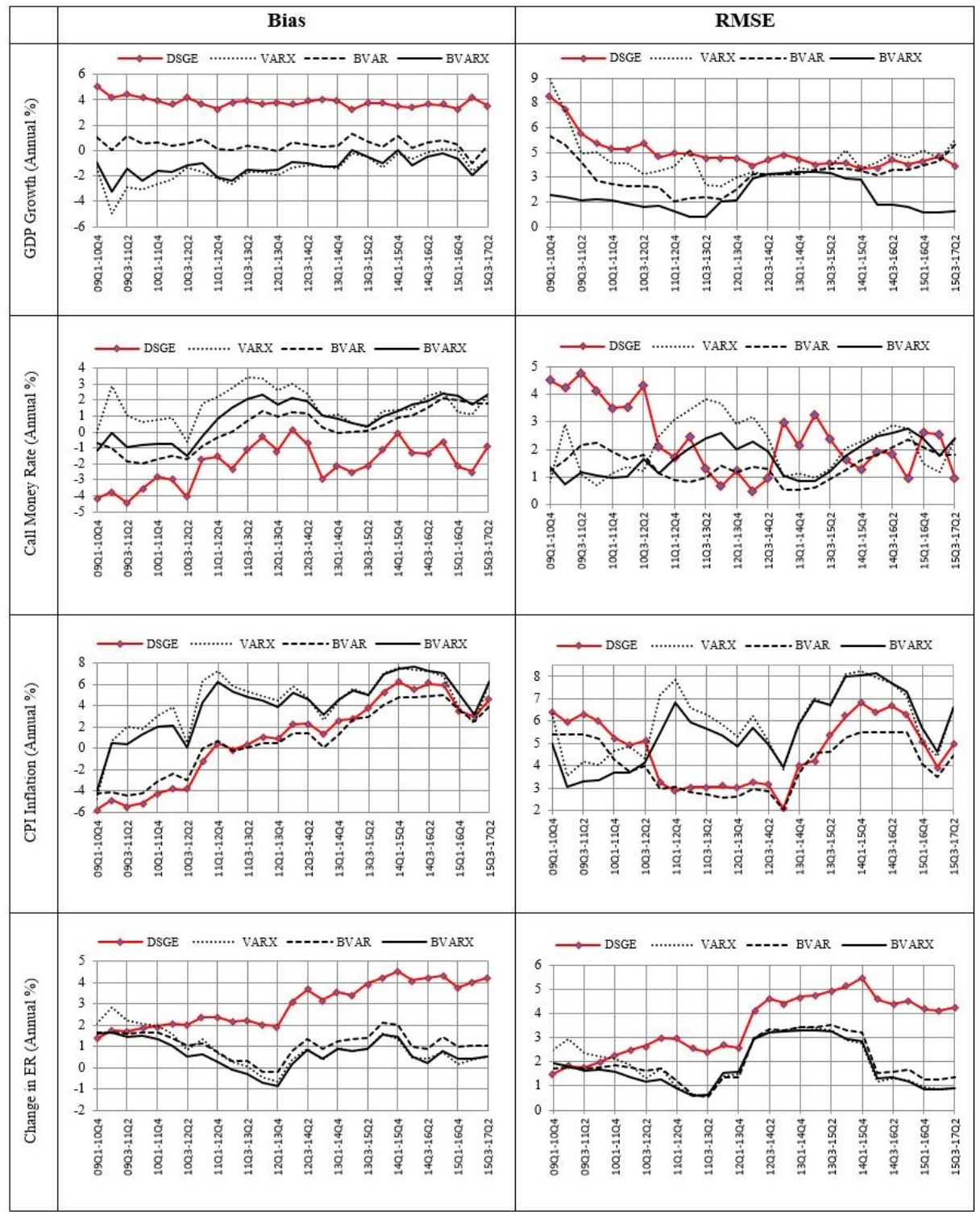

14Q1-16Q3 and 15Q3-17Q2. Structural interpretation of DSGE forecast bias can help evaluate monetary policy stance against the touchstone of DSGE model. Zero bias implies that DSGE model and monetary policy makers are; on average, on the same page. Positive bias implies that actual interest rate is set at a level lower than that suggested by DSGE model and vice versa. It is interesting to note that DSGE forecast depicts negative bias throughout the sample period except for two forecast windows of 12Q2-14Q1 and 14Q1-15Q4. This implies 
that during most of the sample period, SBP's monetary policy stance has been unnecessarily tight; if viewed from the lens of this DSGE model.

Third panel of figure 2 presents bias and RMSE related to annualized quarteron-quarter inflation. During initial sample period of 09Q1-12Q2, BVARX provides relatively better forecast of inflation. After that, BVAR model has dominated during most of the sample period followed by DSGE model that provides RMSE very close to BVAR model. Occasionally, DSGE model even outperforms BVAR. Bias chart confirms that in terms of inflation forecasting, BVAR and DSGE models have similar capabilities.

Fourth panel of figure 2 provides bias and RMSE related to forecasts of quarter-on-quarter percent change in exchange rate. DSGE model provides minimum RMSE during first forecasting window of 09Q1-10Q4. After that, BVARX model provides minimum RMSE forecast throughout the sample period. RMSEs from VARX and BVAR are also very close to BVARX. Bias chart shows that DSGE model consistently over predicts percent change in exchange rate. Structural interpretation of DSGE forecast errors implies that domestic currency has been overvalued during sample period. Existence of large positive bias implies that under given parameterization and theoretical structure, the DSGE model considers domestic currency overvalued. To stay in equilibrium, the model calls for domestic currency depreciation.

\section{Conclusion}

Owing to presence of transmission lag, only forward looking macro policies are expected to successfully achieve their goals. Designing of forward looking policies is not possible without high quality forecasts of relevant macro variables. This study; being a contribution in the area of macroeconomic forecasting, has direct policy implications through improvement in policy making process resulting from better forecasts. Indirect policy implications arise from the fact that DSGE models are general equilibrium models which are capable of addressing a wide range of policy questions. Forecasts from equilibrium models provide trajectories of economic variables where economic system will be in equilibrium during the forecast horizon. So that forecast errors computed from these models can be used to compute gaps i.e. deviations from equilibrium values. For instance, forecast errors in exchange rate, interest rate and output can be used to compute exchange misalignment, interest rate gap and output gap, respectively. These gaps are considered important policy inputs.

Analysis of forecasting performance over 1-8 quarters forecast horizon reveals that BVAR model provides relatively better forecast in case of GDP growth, interest rate and inflation while BVARX provides more accurate forecast in case of exchange rate. In case of GDP growth, inflation and exchange rate, forecasting performance of DSGE model considerably improves as forecasting horizon expands. For longer forecast horizons, divergence between DSGE and Bayesian VAR forecasts tends to disappear. This implies that DSGE model is more relevant for medium term forecasting rather than short term forecasting. 


\section{S. Ahmad, A. Haider}

Analysis of forecasting performance over the sample period 09Q1-17Q2 indicates that BVARX provides relatively better forecast in case of GDP growth and exchange rate. DSGE and BVAR models provide similar forecasting performance in case of call money rate and inflation. Exploiting the general equilibrium nature of DSGE model, we interpret DSGE forecast bias as average deviation between implied equilibrium path and actual value of relevant variables. This exercise reveals that there has been unutilized growth potential in economic activity. This slack in economic activity might be attributable to unnecessarily high interest rate and overvalued exchange rate.

It is important to note that the results related to forecasting performance of our estimated DSGE model cannot be generalized to all DSGE models. A few elements can improve forecasting performance of DSGE models. They include improvement in theoretical structure, provision of better priors and, utilization of more time series during Bayesian estimation process.

\section{References}

Adolfson, M., Laseen, S., Linde, J. and Villani, M. (2005), Bayesian estimation of an open economy dsge model with incomplete pass-through. Working paper, Sveriges Riksbank.

Adolfson, M., Laseen, S., Linde, J. and Villani, M. (2007), 'Bayesian estimation of an open economy dsge model with incomplete pass-through', Journal of International Economics $\mathbf{7 2}(02), 481-511$.

Ahmad, S., Ahmed, W., Khan, S., Pasha, F. and Rehman, M. (2012), Pakistan economy dsge model with informality. Working Paper No. 47, SBP Working Paper Series.

Ahmad, S. and Pasha, F. (2015), 'A pragmatic model for monetary policy analysis i: The case of pakistan', SBP Research Bulletin 11(1), 1-42.

Ahmad, S., Pasha, F. and Rehman, M. (2016), The role of money in explaining business cycles for a developing economy: The case of pakistan. State Bank of Pakistan Working Paper Series.

Ahmed, W., Choudhary, M. A., Khan, S., Naeem, S. and Zoega, G. (2014), 'Determinants of wage stickiness in a developing economy', Economic Modelling 38, 296-304.

Berg, A., Karam, P. D. and Laxton, D. (2006a), A practical model-based approach to monetary policy analysis:overview. IMF Working Paper Series.

Berg, A., Karam, P. D. and Laxton, D. (2006b), Practical model-based monetary policy analysis: A how-to guide. IMF Working Paper Series.

Bloor, C. and Matheson, T. (2011), 'Real-time conditional forecasts with bayesian vars: An application to new zealand', North American Journal of Economics and Finance 22(1), 2642 .

Calvo, G. A. (1983), 'Staggered prices in a utility-maximizing framework', Journal of monetary Economics 12(3), 383-398.

Choudhary, A. and Pasha, F. (2013), The rbc view of pakistan: A declaration of stylized facts and essential models. SBP Working Paper Series.

Choudhary, M. A., Faheem, A., Hanif, M. N., Naeem, S. and Pasha, F. (2016), 'Price setting and price stickiness: A developing economy perspective', Journal of Macroeconomics 48, 44-61.

Choudhri, E. U. and Malik, H. (2012), Monetary policy in pakistan: A dynamic stochastic general equilibrium analysis. International Growth Centre.

Christiano, L. J., Eichenbaum, M. and Evans, C. L. (2005), 'Nominal rigidities and the dynamic effects of a shock to monetary policy', Journal of Political Economy 113(1), 1-45.

Christoffel, K. P., Coenen, G. and Warne, A. (2008), The new area-wide model of the euro area: a micro-founded open-economy model for forecasting and policy analysis. ECB Working Paper Series, 944.

Clarida, R., Gali, J. and Gertler, M. (1999), The science of monetary policy: A new keynesian perspective. Working Paper, 7147, National Bureau of Economic Research. 
Cooley, T. F. and Hansen, G. D. (1989), 'The inflation tax in a real business cycle model', American Economic Review $\mathbf{7 9}(4), 733-748$.

Doan, T., Litterman, R. and Sims, C. (1986), Forecasting and conditional projection using realistic prior distribution. Staff Report 93, Federal Reserve Bank of Minneapolis.

Edge, R. M. and Gurkaynak, R. S. (2010), 'How useful are estimated dsge model forecasts for central bankers?', Brookings Papers on Economic Activity Fall2010, 209-259.

Edge, R. M., Kiley, M. T. and Laforte, J. P. (2010), 'A comparison of forecast performance between federal reserve staff forecasts, simple reduced form models, and a dsge model', Journal of Applied Econometrics 25(4), 720-754.

Erceg, C. J., Henderson, D. W. and Levin, A. T. (2000), 'Optimal monetary policy with staggered wage and price contracts', Journal of monetary Economics 46(2), 281-313.

Haider, A., Din, M. and Ghani, E. (2012), 'Monetary policy, informality and business cycle fluctuations in a developing economy vulnerable to external shocks', Pakistan Development Review 52(4), 609-681.

Haider, A. and Khan, S. U. (2008), 'A small open economy dsge model for pakistan', Pakistan Development Review 47(4), 963-1008.

Hanif, N., Iqbal, J. and Malik, J. (2013), 'Quarterisation of national income accounts of pakistan', SBP Research Bulletin 9(1), 1-61.

Heijdra, B. J. and Ploeg, F. v. d. (2002), Foundations of Modern Macroeconomics, Oxford University Press, Oxford, UK.

Javid, M. and Munir, K. (2010), 'The price puzzle and monetary policy transmission mechanism in pakistan: Structural vector autoregressive approach', Pakistan Development Review 49(4), 449-460.

Litterman, R. B. (1986), 'Forecasting with bayesian vector autoregressions-five years of experience', Journal of Business and Economic Statistics 4(1), 25-38.

Lucas, R. (1976), 'Econometric policy evaluation: A critique', Carnegie-Rochester Conference Series on Public Policy 1(1), 19-46.

Ouliaris, S., Pagan, A. R. and Restrepo, J. (2016), Quantitative macroeconomic modeling with structural vector autoregressions-an eviews implementation. Eviews working paper.

Pagan, A. (2003), Report on the modelling and forecasting at the bank of england. Bank of England, Quarterly Bulletin.

Rehman, M., Khan, S. and Hayat, Z. (2017), A small open economy dsge model with worker's remittance. Working Paper No. 84. State Bank of Pakistan Working Paper Series.

Sims, C. A. (1980), 'Macroeconomics and reality', Econometrica 48(1), 1-48.

Smets, F. and Wouters, R. (2003), 'An estimated dynamic stochastic general equilibrium model of the euro area', Journal of the European Economic Association 1(5), 1123-1175.

Smets, F. and Wouters, R. (2007), 'Shocks and frictions in us business cycles: A bayesian dsge approach', American Economic Review 97(3), 586-606.

Todd, R. (1984), 'Improving economic forecasting with bayesian vector autoregression', Federal Reserve Bank of Minneapolis Quarterly Review 8, 18-29.

Tovar, C. E. (2009), Dsge models and central banks. economics: The open-access. OpenAssessment E-Journal(3)

Wieland, V., Cwik, T., Muller, G. J., Schmidt, S. and Wolters, M. (2012), 'A new comparative approach to macroeconomic modeling and policy analysis', Journal of Economic Behavior and Organization 83(3), 523-541.

\section{Appendices}

\section{A Linearized version of DSGE model}

Following are the final version of linearized equations included in the model.

Business Review: (2019) 14(1):28-52 


\section{A.1 Aggregate supply}

NKPC for domestic inflation:

$$
\begin{aligned}
\left(\hat{\pi}_{t}-\widehat{\bar{\pi}}_{t}^{c}\right)= & \frac{\beta}{1+\kappa_{D} \beta}\left(E_{t} \hat{\pi}_{t+1}-\widehat{\bar{\pi}}_{t}^{c}\right)+\frac{\kappa_{D}}{1+\kappa_{D} \beta}\left(\hat{\pi}_{t-1}-\widehat{\bar{\pi}}_{t}^{c}\right)- \\
& \frac{\kappa_{D} \beta\left(1-\rho_{\pi}\right)}{1+\kappa_{D} \beta} \widehat{\bar{\pi}}_{t}^{c}+\frac{\left(1-\xi_{d}\right)\left(1-\beta \xi_{d}\right)}{\xi_{d}\left(1+\kappa_{D} \beta\right)} \widehat{m c}_{t}
\end{aligned}
$$

Domestic marginal costs:

$$
\widehat{m c}_{t}=\alpha\left(\hat{\mu}_{z, t}+\hat{H}_{t}-\hat{k}_{t}\right)+\widehat{\bar{w}}_{t}+\hat{R}_{t}^{f}-\hat{\epsilon}_{t}
$$

\section{Capital-labour ratio:}

$$
\hat{k}_{t}-\hat{H}_{t}=\hat{\mu}_{z, t}+\hat{R}_{t}^{f}-\hat{r}_{t}^{k}+\widehat{\bar{w}}_{t}
$$

NKPC for imported consumption goods:

$$
\begin{aligned}
\left(\hat{\pi}_{t}^{m, c}-\widehat{\bar{\pi}}_{t}^{c}\right)= & \frac{\beta}{1+\kappa_{m, c} \beta}\left(E_{t} \hat{\pi}_{t+1}^{m, c}-\rho_{\pi} \widehat{\bar{\pi}}_{t}^{c}\right)+\frac{\kappa_{m, c}}{1+\kappa_{m, c} \beta}\left(\hat{\pi}_{t-1}^{m, c}-\widehat{\bar{\pi}}_{t}^{c}\right)- \\
& \frac{\kappa_{m, c} \beta\left(1-\rho_{\pi}\right)}{1+\kappa_{m, c} \beta} \widehat{\widehat{\pi}}_{t}^{c}+\frac{\left(1-\xi_{m, c}\right)\left(1-\beta \xi_{m, c}\right)}{\xi_{m, c}\left(1+\kappa_{m, c} \beta\right)} \widehat{m c}_{t}^{m, c}
\end{aligned}
$$

Imported consumption marginal costs:

$$
\widehat{m c}_{t}^{m, c}=\hat{p}_{t}^{*}+\hat{s}_{t}-\hat{p}_{t}^{m, c}
$$

NKPC for imported investment goods:

$$
\begin{aligned}
\left(\hat{\pi}_{t}^{m, i}-\widehat{\bar{\pi}}_{t}^{c}\right)= & \frac{\beta}{1+\kappa_{m, i} \beta}\left(E_{t} \hat{\pi}_{t+1}^{m, i}-\rho_{\pi} \widehat{\bar{\pi}}_{t}^{i}\right)+\frac{\kappa_{m, i}}{1+\kappa_{m, i} \beta}\left(\hat{\pi}_{t-1}^{m, i}-\widehat{\bar{\pi}}_{t}^{c}\right)- \\
& \frac{\kappa_{m, i} \beta\left(1-\rho_{\pi}\right)}{1+\kappa_{m, i} \beta} \widehat{\bar{\pi}}_{t}^{c}+\frac{\left(1-\xi_{m, i}\right)\left(1-\beta \xi_{m, i}\right)}{\xi_{m, i}\left(1+\kappa_{m, i} \beta\right)}\left(\widehat{m c}_{t}^{m, i}+\hat{\lambda}_{t}^{m, i}\right)
\end{aligned}
$$

Imported investment marginal costs:

$$
\widehat{m c}_{t}^{m, i}=\hat{p}_{t}^{*}+\hat{s}_{t}-\hat{p}_{t}^{m, i}
$$

NKPC for export goods:

$$
\begin{aligned}
\left(\hat{\pi}_{t}^{x}-\widehat{\bar{\pi}}_{t}^{c}\right)= & \frac{\beta}{1+\kappa_{x} \beta}\left(E_{t} \hat{\pi}_{t+1}^{x}-\rho_{\pi} \widehat{\bar{\pi}}_{t}^{c}\right)+\frac{\kappa_{x}}{1+\kappa_{x} \beta}\left(\hat{\pi}_{t-1}^{x}-\widehat{\bar{\pi}}_{t}^{c}\right)- \\
& \frac{\kappa_{x} \beta\left(1-\rho_{\pi}\right)}{1+\kappa_{x} \beta} \widehat{\bar{\pi}}_{t}^{c}+\frac{\left(1-\xi_{x}\right)\left(1-\beta \xi_{x}\right)}{\xi_{x}\left(1+\kappa_{x} \beta\right)} \widehat{m c}_{t}^{x}
\end{aligned}
$$

Marginal costs for export goods:

$$
\widehat{m c}_{t}^{x}=\hat{p}_{t}-\hat{s}_{t}-\hat{p}_{t}^{x}
$$

Wage inflation:

$$
\begin{gathered}
\hat{R}_{t}^{f}=\frac{\nu R}{\nu R+1-\nu} \hat{R}_{t-1} \\
E_{t}\left[\begin{array}{c}
\eta_{0} \widehat{\bar{w}}_{t-1}+\eta_{1} \widehat{\bar{w}}_{t}+\eta_{2} \widehat{\bar{w}}_{t+1}+\eta_{3}\left(\hat{\pi}_{t}^{d}-\widehat{\bar{\pi}}_{t}^{c}\right)+\eta_{4}\left(\hat{\pi}_{t+1}^{d}-\rho_{\widehat{\bar{\pi}}^{c}} \widehat{\bar{\pi}}_{t}^{c}\right)+ \\
\eta_{5}\left(\hat{\pi}_{t-1}^{d}-\widehat{\widehat{\pi}}_{t}^{c}\right)+\eta_{6}\left(\hat{\pi}_{t}^{d}-\rho_{\widehat{\bar{\pi}}^{c}} \widehat{\widehat{\pi}}_{t}^{c}\right)+\eta_{7} \hat{\psi}_{z, t}^{\tau}+\eta_{8} \hat{H}_{t}+\eta_{9} \hat{\tau}_{t}^{y}+\eta_{10} \hat{\tau}_{t}^{w}+\eta_{11} \zeta_{t}^{h}
\end{array}\right]=0
\end{gathered}
$$




\section{A.2 Aggregate demand}

\section{Consumption Euler equation:}

$$
E_{t}\left[\begin{array}{c}
-\beta b \mu_{z} \hat{c}_{t+1}+\left(\mu_{z}^{2}+\beta b^{2}\right) \hat{c}_{t}-b \mu_{z} \hat{c}_{t-1}+b \mu_{z}\left(\hat{\mu}_{z, t}-\beta \hat{\mu}_{z, t+1}\right)+ \\
\left(\mu_{z}-\beta b\right)\left(\mu_{z}-b\right) \hat{\psi}_{z, t+1}+\frac{\tau^{c}}{1+\tau^{c}}\left(\mu_{z}-\beta b\right)\left(\mu_{z}-b\right) \hat{\tau}_{t}^{c}+ \\
\left(\mu_{z}-\beta b\right)\left(\mu_{z}-b\right) \hat{\gamma}_{t}^{c, d}-\left(\mu_{z}-b\right)\left(\mu_{z} \hat{\varsigma}_{t}^{c}-\beta b \hat{\varsigma}_{t+1}^{c}\right)
\end{array}\right]=0
$$

Derivative w.r.t. money:

$E_{t}\left[-\mu \hat{\psi}_{z, t}+\mu \hat{\psi}_{z, t+1}-\mu \hat{\mu}_{z, t+1}+\left(\mu-\beta \tau^{k}\right) \hat{R}_{t}-\mu \hat{\pi}_{t+1}+\frac{\tau^{k}}{1-\tau^{k}}(\beta-\mu) \hat{\tau}_{t+1}^{k}\right]=0$

Money growth:

$$
\mu_{t}=\widehat{\bar{m}}_{t+1}+\hat{\mu}_{z, t}+\hat{\pi}_{t}
$$

Derivative w.r.t. capital:

$$
E_{t}\left[\begin{array}{c}
\hat{\psi}_{z, t}+\hat{\mu}_{z, t+1}-\hat{\psi}_{z, t+1}-\frac{\beta(1-\delta)}{\mu_{z}} \hat{P}_{k^{\prime}, t+1}+\hat{P}_{k^{\prime}, t} \\
-\frac{\mu_{z}-\beta(1-\delta)}{\mu_{z}} \hat{r}_{t+1}^{k}+\frac{\tau^{k}}{1-\tau^{k}} \frac{\mu_{z}-\beta(1-\delta)}{\mu_{z}} \hat{\tau}_{t+1}^{k}
\end{array}\right]=0
$$

Derivative w.r.t. investment:

$$
E_{t}\left[P_{k^{\prime}, t}+\hat{\Upsilon}_{t}-\hat{\gamma}_{t}^{i, d}-\mu_{z}^{2} \tilde{S}^{\prime \prime}\left[\left(\hat{\imath}_{t}-\hat{\imath}_{t-1}\right)-\beta\left(\hat{\imath}_{t+1}-\hat{\imath}_{t}\right)+\hat{\mu}_{z, t}-\beta \hat{\mu}_{z, t+1}\right]\right]=0
$$

Derivative w.r.t. rate of capital utilization:

$$
\hat{u}_{t}=\frac{1}{\sigma} \hat{r}_{t}^{k}-\frac{1}{\sigma_{a}} \frac{\tau^{k}}{1-\tau^{k}} \hat{\tau}_{t}^{k}
$$

Definition of variable capacity utilization:

$$
\hat{u}_{t}=\hat{k}_{t}-\widehat{\bar{k}}_{t}
$$

UIP condition:

$$
E_{t} \Delta \hat{S}_{t+1}-\left(\hat{R}_{t}-\hat{R}_{t}^{*}\right)-\tilde{\phi}_{a} \hat{a}_{t}+\widehat{\tilde{\phi}}_{t}=0
$$

Derivative w.r.t. cash holding:

$$
\hat{q}_{t}=\frac{1}{\sigma_{q}}\left[\hat{\varsigma}_{t}^{q}+\frac{\tau^{k}}{1-\tau^{k}} \hat{\tau}_{t}^{k}-\hat{\psi}_{z, t}-\frac{R}{1-R} \hat{R}_{t}\right]
$$

Business Review: (2019) 14(1):28-52 


\section{A.3 Government and Central Bank}

$$
\hat{R}_{t}=\rho_{R} \hat{R}_{t-1}+\left(1-\rho_{R}\right)\left(\hat{\bar{\pi}}_{t}^{c}+\rho_{\pi}\left(\hat{\pi}_{t}^{c}-\hat{\bar{\pi}}_{t}^{c}\right)+\rho_{y} \hat{y}_{t-1}\right)+\varepsilon_{R, t}
$$

\section{CPI inflation:}

$$
\widehat{\pi}_{t}^{c}=\left[\left(1-\omega_{c}\right)\left(\gamma^{d, c}\right)^{\left(1-\eta_{c}\right)}\right] \hat{\pi}_{t}^{d}+\left[\omega_{c}\left(\gamma^{m c, c}\right)^{\left(1-\eta_{c}\right)}\right] \hat{\pi}_{t}^{m, c}
$$

Production function:

$$
\hat{y}_{t}=\lambda_{d}\left[\hat{\epsilon}_{t}+\alpha \hat{K}_{t}+(1-\alpha) \hat{H}_{t}\right]
$$

Exchange rate gap:

$$
\begin{gathered}
\hat{x}_{t}=-\omega_{c}\left(\gamma^{m c, c}\right)^{-\left(1-\eta_{c}\right)} \gamma^{m c, d}-\hat{\gamma}_{t}^{x, *}-\widehat{m c} c_{t}^{x} \\
\hat{x}_{t}=\hat{S}_{t}+\hat{P}_{t}^{*}-\hat{P}_{t}^{c}
\end{gathered}
$$

Aggregate resource constraint:

$$
\begin{gathered}
\left(1-\omega_{c}\right)\left(\gamma^{c, d}\right)^{\eta_{c}} \frac{c}{y}\left(c_{t}+\eta_{c} \hat{\gamma}_{t}^{c, d}\right)+\left(1-\omega_{i}\right)\left(\gamma^{i, d}\right)^{\eta_{i}} \frac{i}{y}\left(i_{t}+\eta_{i} \hat{\gamma}_{t}^{i, d}\right)+\frac{g}{y} \hat{g}_{t}+ \\
\frac{y^{*}}{y}\left(y_{t}^{*}-\eta_{f} \hat{\gamma}_{t}^{x, *}+\widehat{\widetilde{z}}_{t}^{*}\right)=\lambda_{d}\left(\hat{\epsilon}_{t}+\alpha\left(\hat{k}_{t-} \hat{\mu}_{z, t}\right)+(1-\alpha) \hat{H}_{t}\right)-\left(1-\tau^{k}\right) r^{k} \frac{\bar{k}}{y} \frac{1}{\mu_{z}}\left(\hat{k}_{t}-\hat{\bar{k}}_{t}\right)
\end{gathered}
$$

Capital accumulation constraint:

$$
\widehat{\bar{k}}_{t+1}=(1-\delta) \frac{1}{\mu_{z}}\left(\widehat{\bar{k}}_{t}-\hat{\mu}_{z, t}\right)+\left(1-(1-\delta) \frac{1}{\mu_{z}}\right)\left(\hat{\imath}_{t}+\hat{\Upsilon}_{t}\right)
$$

Net foreign assets:

$$
\begin{aligned}
\hat{a}_{t}= & -y^{*} \widehat{m c}{ }_{t}^{x}-\eta_{f} y^{*} \hat{\gamma}_{t}^{x, *}+y^{*} \hat{y}_{t}^{*}+y^{*} \widehat{\widetilde{z}}_{t}^{*}+\left(c^{m}+i^{m}\right) \hat{\gamma}_{t}^{f}- \\
& c^{m}\left(-\eta_{c}\left(1-\omega_{c}\right)\left(\gamma^{c, d}\right)^{-\left(1-\eta_{c}\right)} \hat{\gamma}_{t}^{m c, d}+\hat{c}_{t}\right)+ \\
& i^{m}\left(-\eta_{i}\left(1-\omega_{i}\right)\left(\gamma^{i, d}\right)^{-\left(1-\eta_{i}\right)} \hat{\gamma}_{t}^{m i, d}+\hat{\imath}_{t}\right)+\frac{R}{\pi \mu_{z}} \hat{a}_{t-1}
\end{aligned}
$$

Loan market clearing condition:

$$
\nu \bar{w}_{t} H\left(\hat{w}_{t}+\hat{H}_{t}\right)=\frac{\mu \bar{m}}{\mu_{z} \pi}\left(\hat{\mu}_{t}+\widehat{\bar{m}}_{t}-\hat{\pi}_{t}-\hat{\mu}_{z, t}\right)
$$

Relative prices:

$$
\begin{gathered}
\hat{\gamma}_{t}^{m c, d}=\hat{\gamma}_{t-1}^{m c, d}+\pi_{t}^{m, c}-\pi_{t}^{d} \\
\hat{\gamma}_{t}^{m i, d}=\hat{\gamma}_{t-1}^{m i, d}+\pi_{t}^{m, i}-\pi_{t}^{d} \\
\hat{\gamma}_{t}^{x, *}=\hat{\gamma}_{t-1}^{x, *}+\pi_{t}^{x}-\pi_{t}^{*} \\
\widehat{m c}_{t}^{x}=\widehat{m c}_{t-1}^{x}+\pi_{t}-\pi_{t}^{x}-\Delta \hat{S}_{t} \\
\hat{\gamma}_{t}^{f}=\widehat{m c}_{t}^{x}+\hat{\gamma}_{t}^{x, *} \\
\hat{\gamma}_{t}^{c, d}=\omega_{c} \gamma^{m c, c\left(1-\eta_{c}\right)} \hat{\gamma}_{t}^{m c, d} \\
\hat{\gamma}_{t}^{i, d}=\omega_{i} \gamma^{m i, i\left(1-\eta_{i}\right)} \hat{\gamma}_{t}^{m i, d}
\end{gathered}
$$

50 


\section{A.4 Exogenous shocks}

Transitory technology shock:

$$
\hat{\epsilon}_{t}=\rho_{\epsilon} \hat{\epsilon}_{t-1}+\varepsilon_{t}
$$

FX risk-premium shock:

$$
\widehat{\tilde{\phi}}_{t}=\rho_{\widetilde{\tilde{\phi}}} \widehat{\tilde{\phi}}_{t-1}+\varepsilon \varepsilon_{\tilde{\phi}, t}
$$

Fiscal spending shock:

$$
\hat{g}_{t}=\rho_{\hat{g}} \hat{g}_{t-1}+\epsilon_{t}^{\hat{g}}
$$

Foreign output:

$$
y_{t}^{*}=\rho_{y^{*}} y_{t-1}^{*}+\varepsilon_{y^{*}, t}
$$

Foreign inflation:

$$
\pi_{t}^{*}=\rho_{\pi^{*}} \pi_{t-1}^{*}+\varepsilon_{\pi^{*}, t}
$$

Foreign interest rate:

$$
R_{t}^{*}=\rho_{R^{*}} R_{t-1}^{*}+\varepsilon_{R^{*}, t}
$$




\section{B Data and empirical results}

Table B.1: Data definitions and sources

\begin{tabular}{clll}
\hline Sr. \# & Data Series Description & Unit & Source \\
\hline 1 & Real GDP (fc) Base=FY2000 & Million PKR & Hanif et al. (2013) \\
2 & Exchange Rate & PKR/USD & SBP \\
3 & CPI & Index & SBP \\
4 & Pakistan and US Population & Million & UN \\
5 & Call Money Rate & Annual \% & SBP \\
6 & USA Real GDP & Billion USD & IFS IMF \\
7 & USA 3-Months T-Bill Rate & Annual \% & IFS IMF \\
8 & USA CPI & Index & IFS IMF \\
\hline
\end{tabular}

Table B.2: Unit root tests

\begin{tabular}{clcc}
\hline Variable & Exogenous Variable & t-statistic & MacKinnon (1996) one-sided p-value \\
\hline$\Delta G D P_{t}$ & Constant & -17.009 & 0.000 \\
$\pi_{t}$ & Constant & -6.421 & 0.000 \\
$\Delta S_{t}$ & Constant & -8.586 & 0.000 \\
$R_{t}$ & Constant & -2.294 & 0.175 \\
$\Delta G D P_{t}^{U S A}$ & Constant & -5.317 & 0.000 \\
$\pi_{t}^{U S A}$ & Constant & -6.925 & 0.000 \\
$R_{t}^{U S A}$ & Constant & -2.874 & 0.051 \\
\hline
\end{tabular}

Table B.3: VAR lag length selection

(* indicates lag order selected by the criterion)

\begin{tabular}{ccccccc}
\hline Lag & LogL & LR & FPE & AIC & SC & HQ \\
\hline 0 & -1474.52 & NA & 28291.79 & 21.6 & 21.94 & 21.74 \\
1 & -1370.6 & 195.8 & 7915.02 & 20.33 & $21.01^{*}$ & $20.60^{*}$ \\
2 & -1358.66 & 21.8 & 8405.63 & 20.39 & 21.4 & 20.8 \\
3 & -1341.23 & 30.81 & 8253.48 & 20.37 & 21.72 & 20.92 \\
4 & -1321.28 & $34.12^{*}$ & $7825.74^{*}$ & $20.31^{*}$ & 22.01 & 21 \\
\hline
\end{tabular}

